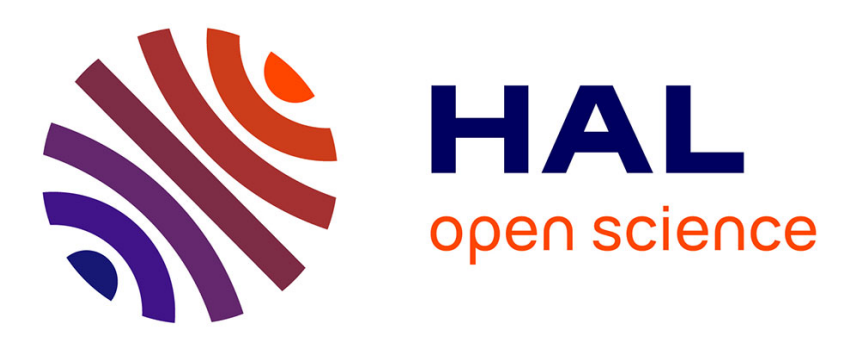

\title{
Electromagnetic force density and energymomentum tensor in an arbitrary continuous medium
}

A Shevchenko, M Kaivola

\section{To cite this version:}

A Shevchenko, M Kaivola. Electromagnetic force density and energymomentum tensor in an arbitrary continuous medium. Journal of Physics B: Atomic, Molecular and Optical Physics, 2011, 44 (17), pp.175401. 10.1088/0953-4075/44/17/175401 . hal-00651666

\section{HAL Id: hal-00651666 https://hal.science/hal-00651666}

Submitted on 14 Dec 2011

HAL is a multi-disciplinary open access archive for the deposit and dissemination of scientific research documents, whether they are published or not. The documents may come from teaching and research institutions in France or abroad, or from public or private research centers.
L'archive ouverte pluridisciplinaire HAL, est destinée au dépôt et à la diffusion de documents scientifiques de niveau recherche, publiés ou non, émanant des établissements d'enseignement et de recherche français ou étrangers, des laboratoires publics ou privés. 


\title{
Electromagnetic force density and energy-momentum tensor in an arbitrary continuous medium
}

\author{
A Shevchenko, M. Kaivola \\ Aalto University, Department of Applied Physics, P.O. Box 13500, FI-00076 AALTO, \\ Finland \\ E-mail: andriy.shevchenko@aalto.fi
}

\begin{abstract}
For more than a century, physicists have searched for a unique and general form for the force density that an electromagnetic field imposes on a medium. The existing equations for this quantity, obtained, e.g., by Minkowski, Einstein and Laub, Abraham, and Helmholtz, are manifestly different and, as such, give different predictions in some particular physical situations. The cases of real media with dispersion, dissipation, and nonlinearities as well as materials with optical gain have not been much addressed. Here we present an unambiguous general equation for the force density expressed in terms of a new energy-momentum tensor of the field. The equation can be applied to almost any natural or designed material. We show how the results obtained by Minkowski, Helmholtz, Einstein and Laub, and Abraham appear as special cases of this general treatment. Surprisingly, our result turns out to be physically very close to the almost forgotten result of Einstein and Laub.
\end{abstract}

PACS numbers: 42.25.Bs, 03.50.De, 75.80.+q, 77.65.-j 


\section{Introduction}

The electric and magnetic field find their physical meaning through the force effects they have on a unit electric charge and current. The force exerted by an electric field $\mathbf{e}$ and a magnetic field $\mathbf{b}$ on a charge $q$ moving with velocity $\mathbf{v}$ is given by the Lorentz equation $\mathbf{F}_{\mathrm{L}}=q \mathbf{e}+q \mathbf{v} \times \mathbf{b}$. This equation is valid also for charges belonging to a continuous medium, in which case, however, $\mathbf{e}$ and $\mathbf{b}$ must be treated as microscopic fields. We recall that the macroscopic fields $\mathbf{E}$ and $\mathbf{B}$ are spatial averages of $\mathbf{e}$ and $\mathbf{b}$, and that the physical meanings of these two pairs are different. In contrast to $\mathbf{e}$ and $\mathbf{b}$, the fields $\mathbf{E}$ and $\mathbf{B}$ can usually readily be calculated, even for complicated geometries, e.g., by solving the macroscopic Maxwell's equations using numerical methods. Thus, if there would exist a general equation for the electromagnetic force density in terms of $\mathbf{E}$ and $\mathbf{B}$, one could insert the calculated $\mathbf{E}$ and $\mathbf{B}$ into this equation and obtain the electromagnetic force density inside any material. Unfortunately, there exist more than one equation for this quantity, and which of them is correct or more general, still remains an open and intensively discussed question [1]-[18].

Among the many existing equations for the electromagnetic force density in a medium, the four most famous ones are those by Minkowski [19], Abraham [20], Einstein and Laub [21], and Helmholtz [1, 22]. These equations yield both quantitatively and qualitatively different results in some particular cases [1]. For example, for a stationary field inside a homogeneous and isotropic medium, both Minkowski's and Abraham's force densities are equal to zero independently of the spatial distribution of the field. In contrast, the Einstein-Laub and Helmholtz equations yield a non-zero force density that compresses the medium towards regions of higher intensity. Furthermore, for a stationary electromagnetic field with vectors $\mathbf{E}$ and $\mathbf{B}$ parallel to a boundary between, say, vacuum and an isotropic dielectric, the force applied to the boundary is (i) equal to zero in the Einstein-Laub picture, (ii) directed away from the medium in both Minkowski's and Abraham's pictures, and (iii) directed into the medium in the Helmholtz picture. As pointed out by Brevik in Ref. [1], the Minkowski, Abraham, and Einstein-Laub results show disagreement at least with the experiments by Hakim and Higham [23] in which they accurately measured the dependence of the hydrostatic pressure in a liquid on the strength of the applied static electric field. Brevik has also underlined the fact that, for stationary fields, the Helmholtz force density is the only one that has not been contradicted in any experiment. To our knowledge, this statement still holds true. However, the Helmholtz theory was originally written for static fields only [22]. Hence, the force density in the theory does not contain any momentum density of the field. Furthermore, the Helmholtz tensor, which later was generalized to account also for oscillating fields [1], is valid only for isotropic and lossless media. In addition, it can be used in practise only if the density derivatives of the electric permittivity and magnetic permeability of the material are known at the frequency of the applied field (as is the case, e.g., for Clausius-Mossotti or Lorentz-Lorenz type media).

In this work, we introduce a general expression for the electromagnetic force 
density written in terms of the three-dimensional energy-momentum tensor and the momentum density of the field for an arbitrary continuous medium. This expression is unambiguous and has a clear physical meaning. In the limit of a linear, isotropic, and non-dispersive material, the obtained tensor and momentum density converge to our earlier expressions [24, 25]. If, in addition, the medium obeys the Clausius-Mossotti law, the derived tensor becomes the Helmholtz tensor. The general equation, however, can be applied also to nonlinear, anisotropic, dispersive, and dissipative media, and even to materials with optical gain. It naturally includes the effects of electro- and magnetostriction. We also calculate the rate of work done by the field on the medium per unit volume, starting from a microscopic picture of the interaction. This rate is known to determine the temporal component of the four-force density. Therefore, it also yields the four-dimensional energy-momentum tensor in space-time. The obtained tensor is compared with those of Minkowski, Abraham, Einstein-Laub, and Helmholtz. In particular, this tensor turns out to be close in its physical content to the EinsteinLaub tensor.

\section{Three-dimensional force density and energy-momentum tensor}

In Ref. [24], by spatially averaging the microscopic Lorentz force density, we derived an expression for the macroscopic force density imposed by an electromagnetic field on a medium in the following form

$$
\mathbf{f}=\rho \mathbf{E}+\mathbf{J} \times \mathbf{B}+\sum_{k=x, y, z}\left(P_{k} \nabla E_{\mathrm{ext}, k}+M_{k} \nabla B_{\mathrm{ext}, k}\right)+\frac{\mathrm{d}}{\mathrm{d} t}\left(\mathbf{P} \times \mathbf{B}_{\mathrm{ext}}\right) \cdot(1)
$$

Here $\rho$ and $\mathbf{J}$ are the macroscopic free electric charge and current densities, respectively. The quantities with subindex $k$ are the projections of the corresponding vector quantities onto the Cartesian coordinate axes. The fields $\mathbf{E}_{\text {ext }} \equiv\left\langle\mathbf{e}_{\text {ext }}(\mathbf{r})\right\rangle_{\delta V}$ and $\mathbf{B}_{\text {ext }} \equiv\left\langle\mathbf{b}_{\text {ext }}(\mathbf{r})\right\rangle_{\delta V}$ are the spatially averaged microscopic electric and magnetic field, respectively, created by all sources that are external to the averaging volume $\delta V$ [24]. These fields are equal to the ordinary macroscopic fields $\mathbf{E}$ and $\mathbf{B}$ from which the selffields of the electric charges that belong to $\delta V$ are excluded. Hence, $\mathbf{E}_{\text {ext }}$ and $\mathbf{B}_{\text {ext }}$ would be the traditional local fields if $\delta V$ would be the volume occupied by a single atom or molecule in the medium. Since $\mathbf{E}_{\text {ext }}$ and $\mathbf{B}_{\text {ext }}$ are averaged over a volume that contains a large number of particles, we can call them the macroscopic local fields. The quantities $\mathbf{P}$ and $\mathbf{M}$ are the macroscopic electric polarization and magnetization, respectively.

From the derivation of Eq. (1) it is evident that this equation is quite general, valid for inhomogeneous, electrically conducting, magnetic, anisotropic, nonlinear, dispersive, and dissipative materials as long as $\mathbf{P}$ and $\mathbf{M}$ are not explicitly written in terms of $\mathbf{E}$ and $\mathbf{H}$. For example, for dispersive materials, $\mathbf{P}$ and $\mathbf{M}$ are related to $\mathbf{E}$ and $\mathbf{H}$ via the wellknown convolution integrals. The general relations connecting the electric displacement $\mathbf{D}$ with $\mathbf{P}$ and $\mathbf{E}$ and the magnetic field $\mathbf{H}$ with $\mathbf{M}$ and $\mathbf{B}$ are $\mathbf{D}=\epsilon_{0} \mathbf{E}+\mathbf{P}$ and 
$\mathbf{B}=\mu_{0}(\mathbf{H}+\mathbf{M})$, and the macroscopic Maxwell's equations find their form as

$$
\begin{aligned}
& \nabla \cdot \mathbf{D}=\rho, \\
& \nabla \cdot \mathbf{B}=0, \\
& -\nabla \times \mathbf{E}=\dot{\mathbf{B}}, \\
& \nabla \times \mathbf{H}=\dot{\mathbf{D}}+\mathbf{J},
\end{aligned}
$$

where the dots above $\mathbf{D}$ and $\mathbf{B}$ denote time derivatives.

In Ref. [25] we showed that, in the particular case of a narrow-band or monochromatic field in a dissipative medium, one can obtain a complex-valued force density in the form

$$
\boldsymbol{f}=\sum_{k=x, y, z}\left(P_{k}^{*} \nabla E_{\mathrm{ext}, k}+M_{k}^{*} \nabla B_{\mathrm{ext}, k}\right)+\frac{\mathrm{d}}{\mathrm{d} t}\left(\mathbf{P}^{*} \times \mathbf{B}_{\mathrm{ext}}\right),
$$

where the asterisk stands for complex conjugation. In this equation, terms like $\rho \mathbf{E}$ and $\mathbf{J} \times \mathbf{B}$ are absent, because $\mathbf{P}$ and $\mathbf{M}$ include the contributions also from conduction charges that oscillate and create additional dipole moments. The medium is considered to be electrically neutral and the complex-valued Maxwell's equations to have the same form as Eqs. (2)-(5), but without $\rho$ and $\mathbf{J}$. If the medium is linear and non-dispersive, one can insert the relations

$$
\begin{aligned}
& \mathbf{P}=\epsilon_{0}(\epsilon-1) \mathbf{E} \text { and } \\
& \mathbf{M}=(\mu-1) \mathbf{H}
\end{aligned}
$$

into Eq. (6) and express the force density in terms of the complex relative permittivity $\epsilon$ and permeability $\mu$ [25], which are the most important materials parameters characterizing electromagnetic properties of any medium. However, if the medium is dispersive (while still linear), this cannot be done in the time domain, and one has to resort to Fourier-transform techniques, and treat each frequency component separately. In fact, applying Fourier transformation to Eq. (1) and neglecting dispersion is another way to obtain Eq. (6). In Ref. [25], however, this equation is derived directly in the microscopic interaction picture.

We proceed with the general Eq. (1). The fields $\mathbf{E}_{\text {ext }}$ and $\mathbf{B}_{\text {ext }}$ are given by the following equations $[24,25]$

$$
\begin{aligned}
& \mathbf{E}_{\text {ext }}=\mathbf{E}+\mathbf{P} /\left(3 \epsilon_{0}\right), \\
& \mathbf{B}_{\text {ext }}=\mathbf{B}-2 \mu_{0} \mathbf{M} / 3,
\end{aligned}
$$

which are valid for an arbitrary medium as long as the relations connecting $\mathbf{P}$ and $\mathbf{M}$ to $\mathbf{E}$ and $\mathbf{H}$ are not established. In terms of the outer products $\nabla \mathbf{E}_{\text {ext }}$ and $\nabla \mathbf{B}_{\text {ext }}$, Eq. (1) can be written in an alternative form as

$$
\mathbf{f}=\rho \mathbf{E}+\mathbf{J} \times \mathbf{B}+\nabla \mathbf{E}_{\mathrm{ext}} \cdot \mathbf{P}+\nabla \mathbf{B}_{\mathrm{ext}} \cdot \mathbf{M}+\frac{\mathrm{d}}{\mathrm{d} t}\left(\mathbf{P} \times \mathbf{B}_{\mathrm{ext}}\right) .
$$

Substituting Eqs. (9) and (10) into Eq. (11), we can write

$$
\mathbf{f}=\mathbf{f}_{e}+\mathbf{f}_{m}+\frac{\mathrm{d}}{\mathrm{d} t} \mathbf{g}
$$


where $\mathbf{f}$ is formally divided into three separate terms

$$
\begin{aligned}
& \mathbf{f}_{\mathrm{e}}=\rho \mathbf{E}+\nabla \mathbf{E} \cdot \mathbf{P}+\nabla \mathbf{P} \cdot \mathbf{P} /\left(3 \epsilon_{0}\right), \\
& \mathbf{f}_{\mathrm{m}}=\mathbf{J} \times \mathbf{B}+\nabla \mathbf{B} \cdot \mathbf{M}-2 \mu_{0} \nabla \mathbf{M} \cdot \mathbf{M} / 3, \text { and } \\
& \mathbf{g}=\frac{\mathrm{d}}{\mathrm{d} t}\left(\mathbf{P} \times \mathbf{B}_{\text {ext }}\right) .
\end{aligned}
$$

In accordance with the law of conservation of energy and linear momentum, Eq. (12) must be writable in the following form

$$
\mathbf{f}=-\nabla \cdot \hat{\mathbf{T}}-\frac{\mathrm{d} \mathbf{G}}{\mathrm{d} t}
$$

where $\hat{\mathbf{T}}$ is the three-dimensional energy-momentum tensor and $\mathbf{G}$ the momentum density of the field in the medium. It is easy to show that Eq. (12) can be cast in this form. For example, to obtain the corresponding transformation for $\mathbf{f}_{e}$ [Eq. (13)], we first write

$$
\nabla \mathbf{P} \cdot \mathbf{P} /\left(3 \epsilon_{0}\right)=\nabla \cdot\left(\frac{P^{2}}{6 \epsilon_{0}} \hat{\mathbf{I}}\right)
$$

where $\hat{\mathbf{I}}$ is the unit tensor and $P$ the magnitude of the polarization vector. Then, using Eq. (2), we find that

$$
\rho \mathbf{E}+\nabla \mathbf{E} \cdot \mathbf{P}=\nabla \cdot(\mathbf{D E})-\frac{\epsilon_{0}}{2} \nabla \cdot\left(E^{2} \hat{\mathbf{I}}\right)+\mathbf{D} \times(\nabla \times \mathbf{E})
$$

Substituting Eqs. (17) and (18) into Eq. (13) and using Eq. (4), we find

$$
\mathbf{f}_{\mathrm{e}}=-\nabla \cdot\left(-\mathbf{D E}+\frac{3 \epsilon_{0} E^{2}-P^{2} / \epsilon_{0}}{6} \hat{\mathbf{I}}\right)-\mathbf{D} \times \dot{\mathbf{B}}
$$

In a similar way Eq. (14) can be rewritten to take on the form

$$
\mathbf{f}_{\mathrm{m}}=-\nabla \cdot\left(-\mathbf{B H}+\frac{3 \mu_{0} H^{2}-\mu_{0} M^{2}}{6} \hat{\mathbf{I}}\right)-\dot{\mathbf{D}} \times \mathbf{B}
$$

Finally, substitution of Eqs. (19) and (20) into Eq. (12) yields $\mathbf{f}$ in the form of Eq. (16) with $\hat{\mathbf{T}}$ and $\mathbf{G}$ given by

$$
\begin{aligned}
\hat{\mathbf{T}}= & -\mathbf{D E}+\frac{3 \epsilon_{0} E^{2}-P^{2} / \epsilon_{0}}{6} \hat{\mathbf{I}} \\
& -\mathbf{B H}+\frac{3 \mu_{0} H^{2}-\mu_{0} M^{2}}{6} \hat{\mathbf{I}}, \\
\mathbf{G}= & \mathbf{D} \times \mathbf{B}-\mu_{0} \mathbf{P} \times \mathbf{H}-\frac{\mu_{0}}{3} \mathbf{P} \times \mathbf{M} .
\end{aligned}
$$

These simple equations are the main result of this work. In Refs. [24] and [25], the expressions for $\hat{\mathbf{T}}$ and $\mathbf{G}$ were derived for cases in which the dispersion, anisotropy, and nonlinearity of the medium can be ignored. Equations (21) and (22) apply to any medium. They are in full agreement with the results of Refs. [24] and [25]. It can be easily seen that if the medium is not magnetic, so that we can write $\mathbf{M}=0$ and $\mathbf{B}=\mu_{0} \mathbf{H}$, Eq. (22) yields

$$
\mathbf{G}=\frac{1}{c^{2}} \mathbf{E} \times \mathbf{H}
$$

independently of the dielectric properties of the material; $c$ is the speed of light in vacuum. 


\section{Physical interpretation}

To gain physical insight into Eqs. (21) and (22), we write them in the form

$$
\begin{aligned}
\hat{\mathbf{T}}= & -\mathbf{D E}+\left(\frac{1}{2} \mathbf{D} \cdot \mathbf{E}+V_{\mathrm{el}}\right) \hat{\mathbf{I}} \\
& -\mathbf{B H}+\left(\frac{1}{2} \mathbf{B} \cdot \mathbf{H}+V_{\mathrm{mag}}\right) \hat{\mathbf{I}}, \\
\mathbf{G}= & \mathbf{D} \times \mathbf{B}-\mathbf{g},
\end{aligned}
$$

where

$$
\begin{aligned}
V_{\mathrm{el}} & =-\frac{1}{2} \mathbf{P} \cdot \mathbf{E}_{\mathrm{ext}} \\
V_{\mathrm{mag}} & =-\frac{1}{2} \mathbf{M} \cdot \mathbf{B}_{\mathrm{ext}}
\end{aligned}
$$

and

$$
\mathbf{g}=\mathbf{P} \times \mathbf{B}_{\mathrm{ext}}
$$

The quantities $V_{\mathrm{el}}$ and $V_{\mathrm{mag}}$, which make the tensor differ from the Minkowski tensor, are the potential energy densities of the medium interacting with the macroscopic electric and magnetic local fields [26, 27]. Consequently, the quantity

$$
V=V_{\mathrm{el}}+V_{\mathrm{mag}}
$$

which is the total density of the field-matter interaction potential, is equal to the density of the work the field has done on the medium (which is also equal to the density of work done in introducing the medium into the field) [27]. Note that, in principle, $V$ can be positive, but at least for static and quasi-monochromatic fields, its time-averaged value is negative. The terms $V_{\mathrm{el}}$ and $V_{\mathrm{mag}}$ are responsible for electro- and magnetostriction, respectively, and lead to electromagnetic forces that compress the medium towards regions of higher intensity.

It is worth mentioning that if Eqs. (26)-(28) would contain the ordinary macroscopic fields $\mathbf{E}$ and $\mathbf{B}$ instead of $\mathbf{E}_{\text {ext }}$ and $\mathbf{B}_{\text {ext }}$, then $\hat{\mathbf{T}}$ and $\mathbf{G}$ would be exactly the energymomentum tensor and the field momentum density in the Einstein-Laub theory $[1,21]$. In the present form, the tensor becomes the Helmholtz tensor, if the medium is a transparent dielectric that obeys the Clausius-Mossotti law [24, 25].

Equation (25) shows that the momentum density of the field is equal to the Minkowski momentum density minus a vector quantity g. In Ref. [28], the quantity $\mathbf{g}$ with $\mathbf{B}$ used instead of $\mathbf{B}_{\text {ext }}$ is referred to as a pseudomomentum density. If the same pseudomomentum density would be used in Eq. (25), G would be exactly the electromagnetic momentum density of Ref. [28]. For non-magnetic materials, $\mathbf{G}$ is indeed the same as in [28], and it is also the same as the one in the Einstein-Laub and Abraham theories [1, 20, 21], i.e., equal to that in vacuum.

Equations (16), (21), and (22) [or alternatively (24) and (25)] can be used to calculate the force density imposed by an electromagnetic field on an arbitrary medium that can be described by the macroscopic Maxwell's equations. This force density will 
govern the motion of the medium and its parts, and lead to deformation and compression of the medium. The force on an arbitrary part of the medium can be calculated by integrating $\mathbf{f}$ over the volume of this part, and any object placed in a homogeneous medium can be considered simply as a part of a general inhomogeneous medium.

Next we discuss some consequences of the presence of $V$ in the equation for $\mathbf{f}$. After the introduction of an electromagnetic field in a medium, the potential $V$ will on average lead to a compression of the medium towards the points of maximum intensity. As a result, the internal pressure of the medium, $p$, will evolve towards an equilibrium at a time scale determined by the speed of sound in the medium [1], starting from the original value $p_{0}$ of the pressure. After reaching an equilibrium, the field-induced excess pressure, $p_{\text {exc }}=p-p_{0}$, will be distributed in accordance to $p_{\text {exc }}=-V$ to compensate for the electromagnetic compression. The unevenly distributed excess pressure will lead to appearance of an Archimedes-like force density

$$
\mathbf{f}_{\mathrm{exc}}=-\nabla p_{\mathrm{exc}}=\nabla V
$$

with which the medium will eventually act on itself. The force density $\mathbf{f}_{\text {exc }}$ is equal in magnitude and opposite in direction to the electromagnetic compressive force density $-\nabla V$. Thus, at equilibrium, the overall force density applied to each point inside the medium is $\widetilde{\mathbf{f}}=\mathbf{f}+\mathbf{f}_{\text {exc. }}$. If the field is harmonic or stationary, the time-averaged force density $\widetilde{\mathbf{f}}$ is equal to the time-averaged force density introduced by Minkowski, i.e.,

$$
\langle\widetilde{\mathbf{f}}\rangle=\left\langle\mathbf{f}_{\mathrm{M}}\right\rangle=-\nabla \cdot\langle\hat{\mathbf{T}}-V \hat{\mathbf{I}}\rangle,
$$

where the fact that $\langle\dot{\mathbf{G}}\rangle=0$ is used. On the other hand, if the field is quasimonochromatic and has slowly varying amplitudes $\mathbf{E}_{0}(t)$ and $\mathbf{B}_{0}(t)$, such that on average the field-matter system evolves adiabatically, then at each point in the medium the force density $\widetilde{\mathbf{f}}$ averaged over one or several oscillation periods is

$$
\langle\widetilde{\mathbf{f}}\rangle=\left\langle\mathbf{f}+\mathbf{f}_{\mathrm{exc}}\right\rangle=-\nabla \cdot\left\langle\hat{\mathbf{T}}_{\mathrm{M}}\right\rangle-\langle\dot{\mathbf{G}}\rangle,
$$

with $\mathbf{G}$ given by Eq. (22). Note that for non-magnetic materials, $\mathbf{G}$ is described by the Abraham's expression and not by the Minkowski's one. Here we want to underline the fact that within a single oscillation period of a high-frequency (say, optical) field, the equilibrium cannot be reached and the results of Minkowski and Abraham cannot be applied. Neither can they be applied to the case of a short electromagnetic pulse (wavepacket), if the pulse's temporal duration is shorter than the time required for sound to transversely cross the pulse.

To summarize, if $\langle\dot{\mathbf{G}}\rangle=0$, Minkowski's tensor describes correctly the total time-averaged force density $\widetilde{\langle\mathbf{f}}\rangle$ in a medium that is already compressed by the field and equilibrated. The same can be said about Abraham's tensor if the medium is isotropic, since in this case the two tensors are equal. This makes it difficult to experimentally resolve the Abraham-Minkowski controversy. We emphasize, however, that the properties of the material, such as volume, density, inhomogeneity, and anisotropy, can change under the compression or deformation by the field. Furthermore, before the system reaches an equilibrium, the excess pressure will change and will not 
compensate for the compressive action of the electromagnetic force density. In such cases, the Minkowski's and Abraham's tensors are insufficient for describing even the time-averaged reaction of the medium to the field.

\section{Rate of work and Poynting theorem}

We proceed to calculating the rate of work done by the field on the medium. Within the concept of four-dimensional space-time, this rate determines the projection of the four-force density onto the temporal axis [26]. Therefore this calculation, in conjunction with Eq. (16), must also yield the four-dimensional energy-momentum tensor.

On the microscopic level, the field-matter interaction is unambiguously described by the microscopic Maxwell's equations

$$
\begin{aligned}
& \epsilon_{0} \nabla \cdot \mathbf{e}=\xi, \\
& \nabla \cdot \mathbf{b}=0, \\
& -\nabla \times \mathbf{e}=\dot{\mathbf{b}}, \\
& \frac{1}{\mu_{0}} \nabla \times \mathbf{b}=\epsilon_{0} \dot{\mathbf{e}}+\mathbf{j},
\end{aligned}
$$

where $\xi$ and $\mathbf{j}$ are the microscopic electric charge and current density, respectively. Within the microscopic picture, the rate of work done by the electromagnetic field on a medium, per unit volume, is given by [26, 27]

$$
\dot{a}=\mathbf{j} \cdot \mathbf{e} .
$$

The electric current density $\mathbf{j}$ can be written in terms of the atomic characteristics as [24]

$$
\mathbf{j}=\sum_{i} \tilde{q}_{i} \dot{\mathbf{r}}_{i} \delta\left(\mathbf{r}-\mathbf{r}_{i}\right)+\sum_{l}\left(\dot{\mathbf{d}}_{l}+\nabla \times \mathbf{m}_{l}\right) \delta\left(\mathbf{r}-\mathbf{r}_{l}\right)
$$

where $\tilde{q}_{i}$ denotes the magnitude of a free charge $i$ located at coordinate $\mathbf{r}_{i}$ in the medium, $\mathbf{d}_{l}$ and $\mathbf{m}_{l}$ are the electric and magnetic dipole moments, respectively, of an atom $l$ located at $\mathbf{r}_{l}$, and $\delta\left(\mathbf{r}-\mathbf{r}_{l}\right)$ is the Dirac delta function centered at $\mathbf{r}_{l}$. Equation (38) holds within the electric and magnetic dipole approximation that is usually used to derive the macroscopic Maxwell's equations from the microscopic ones [24, 29].

The macroscopic rate of work $\dot{A}$ is found in a standard way by spatially averaging $\dot{a}$ over an elementary spherical volume $\delta V$. Using Eqs. (37) and (38), Maxwell's equation (35), and the integration properties of the delta function, we obtain

$$
\dot{A}=\frac{1}{\delta V} \sum_{i \text { in } \delta V} \tilde{q}_{i} \dot{\mathbf{r}}_{i} \cdot \mathbf{e}\left(\mathbf{r}_{i}\right)+\frac{1}{\delta V} \sum_{l \text { in } \delta V}\left(\dot{\mathbf{d}}_{l} \cdot \mathbf{e}\left(\mathbf{r}_{l}\right)+\mathbf{m}_{l} \cdot \dot{\mathbf{b}}\left(\mathbf{r}_{l}\right)\right),
$$

where the summation is performed over the particles located in $\delta V$. Within the small $\delta V$, the free charges and atoms can be considered to be uniformly distributed and have constant characteristics; $\tilde{q}_{i} \dot{\mathbf{r}}_{i}=\tilde{q} \mathbf{v}, \mathbf{d}_{l}=\mathbf{d}$ and $\mathbf{m}_{l}=\mathbf{m}$. This allows us to write

$$
\dot{A}=\frac{1}{\delta V} \tilde{q} \mathbf{v} \cdot \sum_{i \text { in } \delta V} \mathbf{e}\left(\mathbf{r}_{i}\right)+\frac{1}{\delta V}\left(\dot{\mathbf{d}} \cdot \sum_{l \text { in } \delta V} \mathbf{e}\left(\mathbf{r}_{l}\right)+\mathbf{m} \cdot \sum_{l \text { in } \delta V} \dot{\mathbf{b}}\left(\mathbf{r}_{l}\right)\right) .
$$


The sum over the free charge coordinates in the first term can be substituted with $\widetilde{N} \mathbf{E}$ [24], where $\mathbf{E}$ is the volume averaged electric field and $\widetilde{N}$ is the number of free charges in $\delta V$. Note that the volume averaged electric field produced by the charges themselves is equal to zero under the assumption of their uniform distribution in $\delta V$. Since $\tilde{q} \mathbf{v} \widetilde{N} / \delta V=\mathbf{J}$, we have

$$
\dot{A}_{1}=\mathbf{J} \cdot \mathbf{E}
$$

where $\dot{A}_{1}$ stands for the first term in Eq. (40). Before making similar substitutions in the second term, we must exclude the strongly inhomogeneous own fields of the dipoles, $\mathbf{e}_{\text {Own }}$ and $\mathbf{b}_{\text {Own }}$, from $\mathbf{e}\left(\mathbf{r}_{l}\right)$ and $\mathbf{b}\left(\mathbf{r}_{l}\right)$. The field superposition principle allows us to write $\mathbf{e}(\mathbf{r})=\mathbf{e}_{\text {ext }}(\mathbf{r})+\mathbf{e}_{\text {own }}(\mathbf{r})$ and $\mathbf{b}(\mathbf{r})=\mathbf{b}_{\text {ext }}(\mathbf{r})+\mathbf{b}_{\text {own }}(\mathbf{r})$, where $\mathbf{e}_{\text {ext }}(\mathbf{r})$ and $\mathbf{b}_{\text {ext }}(\mathbf{r})$ are the fields produced by all sources external to $\delta V$. For brevity, we do not include the fields of the free charges in these expansions, since they anyway average to zero. In accordance to these expansions, we write for the second term in Eq. (40)

$$
\dot{A}_{2}=\dot{A}_{2} \text {, ext }+\dot{A}_{2} \text {, own } .
$$

We now replace the averaging of the smooth external fields over the dipole coordinates with volume averaging and, using the definitions $\left\langle\mathbf{e}_{\text {ext }}(\mathbf{r})\right\rangle_{\delta V}=\mathbf{E}_{\text {ext }}$ and $\left\langle\mathbf{b}_{\text {ext }}(\mathbf{r})\right\rangle_{\delta V}=$ $\mathbf{B}_{\text {ext }}$, we obtain

$$
\dot{A}_{2, \text { ext }}=\dot{\mathbf{P}} \cdot \mathbf{E}_{\text {ext }}+\mathbf{M} \cdot \dot{\mathbf{B}}_{\text {ext }}
$$

Here $\mathbf{P} \equiv N_{\delta V} \mathbf{d} / \delta V, \mathbf{M} \equiv N_{\delta V} \mathbf{m} / \delta V$, and $N_{\delta V}$ is the number of dipoles in $\delta V$.

When evaluating the term

$$
\dot{A}_{2, \text { own }}=\frac{1}{\delta V}\left(\dot{\mathbf{d}} \cdot \sum_{l \text { in } \delta V} \mathbf{e}_{\text {own }}\left(\mathbf{r}_{l}\right)+\mathbf{m} \cdot \sum_{l \text { in } \delta V} \dot{\mathbf{b}}_{\text {own }}\left(\mathbf{r}_{l}\right)\right),
$$

we depart from the classical picture of a two-point-charge dipole and consider each dipole (atom) as a positively charged nucleus surrounded by a spherical electron shell. This is the simplest model that helps one avoid the problems in describing the dipolefield interaction without resorting to quantum mechanics (e.g., the problem of infinitely growing internal fields and unavoidable collapse of a two-point-charge dipole when in a weakened field the charge separation vanishes). In an external electric field, the shell moves with respect to the nucleus. However, the total field created by the shell is equal to zero at the position of the nucleus, even if it is shifted from the shell center. Similarly, the field produced by the nucleus is equal to zero when integrated over the shell. We can therefore exclude the field of each atom $l$ from $\mathbf{e}_{\text {own }}\left(\mathbf{r}_{l}\right)$ in Eq. (44). The same can be done to $\dot{\mathbf{b}}_{\text {own }}\left(\mathbf{r}_{l}\right)$, since $-\nabla \times \mathbf{e}=\dot{\mathbf{b}}$. This allows us to replace the sums in Eq. (44) with volume integrals, thus replacing the averaging of $\mathbf{e}_{\text {own }}(\mathbf{r})$ and $\mathbf{b}_{\text {Own }}(\mathbf{r})$ over the atomic coordinates with volume averaging. The fields produced by each atom averaged over the volume $\delta V$ from which the volume occupied by the atom itself is excluded (due to the reasons explained above) are equal to zero. So are the sums in Eq. (44), which yields $\dot{A}_{2}$, own $=0$. In principle, the obtained result reflects the known fact that, on 
average, dipoles that compose a continuous medium interact exclusively with the local fields. Using the obtained expressions for $\dot{A}_{1}$ and $\dot{A}_{2}$, ext, we write

$$
\dot{A}=\mathbf{J} \cdot \mathbf{E}+\dot{\mathbf{P}} \cdot \mathbf{E}_{\text {ext }}+\mathbf{M} \cdot \dot{\mathbf{B}}_{\mathrm{ext}} .
$$

With the help of Eqs. (2)-(5) and using Eqs. (9), (10), and (45), we then obtain

$$
\begin{aligned}
\dot{A} & =-\frac{\mathrm{d}}{\mathrm{d} t}\left(\frac{3 \epsilon_{0} E^{2}-P^{2} / \epsilon_{0}}{6}+\frac{3 \mu_{0} H^{2}-\mu_{0} M^{2}}{6}\right) \\
& -\nabla \cdot(\mathbf{E} \times \mathbf{H})+\mathbf{B} \cdot \dot{\mathbf{H}}-\mathbf{H} \cdot \dot{\mathbf{B}} .
\end{aligned}
$$

This equation can be rewritten in a compact and more insightful form as

$$
\dot{A}=-\dot{W}-\dot{V}-\nabla \cdot \mathbf{S}+\zeta
$$

where

$$
\begin{aligned}
& W=\frac{1}{2}(\mathbf{D} \cdot \mathbf{E}+\mathbf{B} \cdot \mathbf{H}), \\
& \mathbf{S}=\mathbf{E} \times \mathbf{H}, \text { and } \\
& \zeta=\mathbf{B} \cdot \dot{\mathbf{H}}-\mathbf{H} \cdot \dot{\mathbf{B}} .
\end{aligned}
$$

In Eqs. (47)-(50), $W$ is a quantity that is usually referred to as the energy density of the field propagating in the medium, $V$ is the interaction potential given by Eq. (29), $\mathbf{S}$ is the Poynting vector that in most cases describes the electromagnetic energy flow, and $\zeta$ is a term that is non-zero only if the medium exhibits magnetic temporal dispersion or magnetic nonlinearity. For usual propagating electromagnetic fields (say, with frequencies above $1 \mathrm{GHz}$ ) in usual materials, the magnetic properties mentioned are absent. It is worth to note that if we move $-\dot{V}$ and $\zeta$ to the left-hand side of Eq. (47) and use Eq. (45), we obtain

$$
\mathbf{J} \cdot \mathbf{E}+\frac{1}{2}(\dot{\mathbf{P}} \cdot \mathbf{E}-\mathbf{P} \cdot \dot{\mathbf{E}})+\frac{1}{2}(\dot{\mathbf{M}} \cdot \mathbf{B}-\mathbf{M} \cdot \dot{\mathbf{B}})=-\dot{W}-\nabla \cdot \mathbf{S}
$$

The pairs $\{\mathbf{P}, \mathbf{E}\}$ and $\{\mathbf{M}, \mathbf{B}\}$ are seen to enter symmetrically in this equation and form expressions similar to Eq. (50). In the absence of temporal dispersion and nonlinearity, both electric and magnetic, Eq. (51) coincides with the differential Poynting theorem in its simple and widely used form $[26,27]$

$$
\mathbf{J} \cdot \mathbf{E}=-\dot{W}-\nabla \cdot \mathbf{S} .
$$

\section{Four-force density and energy-momentum tensor}

Now we can obtain the equation for the four-force density in terms of a $4 \times 4$ energymomentum tensor in space-time for the case of a medium that does not exhibit magnetic dispersion and nonlinearity. The three spatial components of the four-force density are given by Eq. (16). The temporal component is given by $\mathrm{f}_{t}=\dot{A} / c$ [26], where the expression for $\dot{A}$ was introduced in Eq. (47); $\zeta$ is set to zero. Denoting the four-force density by $\mathbf{f}_{4} \equiv\left(\mathrm{f}_{t} \mathrm{f}_{x} \mathrm{f}_{y} \mathrm{f}_{z}\right)^{\top}$, where $\mathrm{T}$ stands for transpose, and the operator nabla by $\nabla_{4} \equiv\left(\frac{1}{c} \mathrm{~d} / \mathrm{d} t \mathrm{~d} / \mathrm{d} x \mathrm{~d} / \mathrm{d} y \mathrm{~d} / \mathrm{d} z\right)^{\top}$, we obtain

$$
\mathbf{f}_{4}=-\nabla_{4} \cdot \hat{\mathbf{T}}_{4}
$$


where

$$
\hat{\mathbf{T}}_{4}=\mathrm{T}^{i j}=\left(\begin{array}{cccc}
W+V & c G_{x} & c G_{y} & c G_{z} \\
S_{x} / c & T_{x x} & T_{x y} & T_{x z} \\
S_{y} / c & T_{y x} & T_{y y} & T_{y z} \\
S_{z} / c & T_{z x} & T_{z y} & T_{z z}
\end{array}\right)
$$

is a four-dimensional contravariant energy-momentum tensor. This tensor can be expanded as

$$
\begin{aligned}
\hat{\mathbf{T}}_{4} & =\left(\begin{array}{cccc}
W & c G_{x}^{(M)} & c G_{y}^{(M)} & c G_{z}^{(M)} \\
S_{x} / c & U_{x x}+W & T_{x y} & T_{x z} \\
S_{y} / c & T_{y x} & U_{y y}+W & T_{y z} \\
S_{z} / c & T_{z x} & T_{z y} & U_{z z}+W
\end{array}\right) \\
& +\left(\begin{array}{cccc}
V & -c g_{x} & -c g_{y} & -c g_{z} \\
0 & V & 0 & 0 \\
0 & 0 & V & 0 \\
0 & 0 & 0 & V
\end{array}\right),
\end{aligned}
$$

where $U_{i i}=-D_{i} E_{i}-B_{i} H_{i}$ and $\mathbf{G}^{(M)}=\mathbf{D} \times \mathbf{B}$. The first term represents the Minkowski tensor that is traceless. The trace of $\hat{\mathbf{T}}_{4}$, that is the sum of the diagonal elements of a mixed tensor $\mathrm{T}_{i}^{j}$, is equal to $-2 V$. If the medium is vacuum or the potential energy density $V$ is uniform over the region of interest, then $V$ is equal to or can be set to be equal to 0 , which makes $\hat{\mathbf{T}}_{4}$ traceless. The trace of the four-dimensional EinsteinLaub tensor is also equal to $-2 V$, where, however, $V$ is written without the local field corrections.

The tensor $\hat{\mathbf{T}}_{4}$ is in general not symmetric, but if the medium is linear, isotropic, non-dissipative, and non-magnetic (so that the intrinsic angular momentum of the field cannot be transferred to the medium), the sum in Eq. (55) is symmetric owing to the offdiagonal elements $-c g_{i}$. So is $\hat{\mathbf{T}}_{4}$ in this case. The asymmetry of $\hat{\mathbf{T}}_{4}$ is connected with the fact that the classical torque density $\mathbf{r} \times \mathbf{f}$ does not contain a contribution from the intrinsic (spin) angular momentum of the field; this contribution to a vector component $l$ of the torque density is given by $T_{k i}-T_{i k}$, where $(i, k, l)$ are cyclic combinations of the indices (see Eq. (4.61) in Ref. [1]).

With regard to the four-dimensional Einstein-Laub tensor we note that among the other mentioned tensors this tensor is indeed very close to ours. Similarly to $\hat{\mathbf{T}}_{4}$, it is neither traceless nor symmetric. It differs from our tensor only in the fields $\mathbf{E}$ and $\mathbf{B}\left[\mathbf{E}_{\text {ext }}\right.$ and $\mathbf{B}_{\text {ext }}$ in our case $]$ in the expressions for $V$ and $\mathbf{g}$. We would also like to mention here the recent theoretical developments by Mansuripur that are in direct correspondence with the Einstein-Laub theory [12].

\section{CONCLUSIONS}

We have introduced a general expression for the macroscopic electromagnetic force density in an arbitrary continuous medium. We have derived the expressions for the 
three-dimensional energy-momentum tensor and the momentum density of the field applicable to any medium. We have discussed the connections of our results with the corresponding quantities in the theories of Minkowski, Helmholtz, Einstein and Laub, and Abraham. Similarly to the Helmholtz tensor, our tensor describes also electro- and magnetostriction effects. It converges to the Helmholtz tensor, if the medium obeys the Clausius-Mossotti law. Thus, our tensor is automatically in agreement with all experiments in which the Helmholtz tensor has been shown to be valid $[1,24]$. New phenomena can be discovered by applying the new Eqs. (21) and (22) to such media that are beyond the applicability of the Helmholtz tensor, i.e., to an anisotropic, nonlinear, dispersive, or dissipative medium as well as to a medium with optical gain [25].

We have shown that, in order to calculate the overall time-averaged force density originating from an electromagnetic field in a medium that is in equilibrium with the field, one can use the Minkowski tensor. In this case, the field-induced compression effects must be properly taken into account. The field momentum density, however, must be described using the Abraham's expression if the medium is not magnetic.

We have also derived the rate of work done by the field on the medium per unit volume. Using the derived expression, we have obtained the four-dimensional energy-momentum tensor in space-time. This tensor is physically very similar to the Einstein-Laub tensor. From a practical point of view, our tensor allows calculating motion, compression, and deformation of a medium by an arbitrary electromagnetic field. We hope that this work will not only contribute to the long-awaited resolution and clarification of the Abraham-Minkowski controversy, but also find useful applications.

\section{Acknowledgments}

We acknowledge financial support from the Academy of Finland (Project 134029) and thank Prof. M. Alonso, University of Rochester, for very useful discussions.

\section{References}

[1] Brevik I 1979 Phys. Rep. 52133.

[2] Leonhardt U 2006 Nature $\mathbf{4 4 4} 823$.

[3] Buchanan M 2007 Nature 373.

[4] Kemp B A, Grzegorczyk T M and Kong J A 2006 Phys. Rev. Lett. 97133902.

[5] Barnett S M and Loudon R 2006 J. Phys. B: At. Mol. Opt. Phys. 39 S671.

[6] Kemp B A, Kong J A and Grzegorczyk T M 2007 Phys. Rev. A 75053810.

[7] She W, Yu J and Feng R 2008 Phys. Rev. Lett. 101243601.

[8] Pfeifer R N C, Nieminen T A, Heckenberg N R and Rubinsztein-Dunlop H 2009 Phys. Rev. A 79 023813.

[9] Hinds E A and Barnett S M 2009 Phys. Rev. Lett. 102050403.

[10] Brevik I 2009 Phys. Rev. Lett. 103219301.

[11] Brevik I and Ellingsen S A 2010 Phys. Rev. A 81 011806(R).

[12] Mansuripur M 2007 Opt. Express 15 13502; 2008 Opt. Express 16 5193; 2010 Opt. Commun. 283 1997.

[13] Barnett S M 2010 Phys. Rev. Lett. 104070401. 
[14] Milonni P W and Boyd R W 2010 Adv. Opt. Photon. 2519.

[15] Baxter C and Loudon R 2010 J. Mod. Opt. 57830.

[16] Barnett S M and Loudon R 2010 Phil. Trans. R. Soc. A 368927.

[17] Brevik I and Ellingsen S Å 2011 Ann. Phys. 326754.

[18] Kemp B A 2011 J. Appl. Phys. 10911101.

[19] Minkowski H 1908 Nachr. Ges. Wiss. Göttingen 53; 1910 Math. Ann. 68472.

[20] Abraham M 1909 Rend. Circ. Matem. Palermo 281.

[21] Einstein A and Laub J 1908 Ann. Physik 26541.

[22] von Helmholtz H 1881 Wied. Ann. 13385.

[23] Hakim S S and Higham J B 1962 Proc. Phys. Soc. 80190.

[24] Shevchenko A, Hoenders B J 2010 New J. Phys. 12053020.

[25] Shevchenko A, Kaivola M 2011 J. Phys. B: At. Mol. Opt. Phys. 44065403.

[26] Jackson J D 1975 Classical Electrodynamics (John Wiley \& Sons, New York).

[27] Straton J A 1941 Electromagnetic theory (McGraw-Hill Book Company, New York and London).

[28] Loudon R, Allen L, Nelson D F 1997 Phys. Rev. E 551071.

[29] Russakoff G 1970 Am. J. Phys. 381188. 